\title{
«If we do not build it other people will!»
}

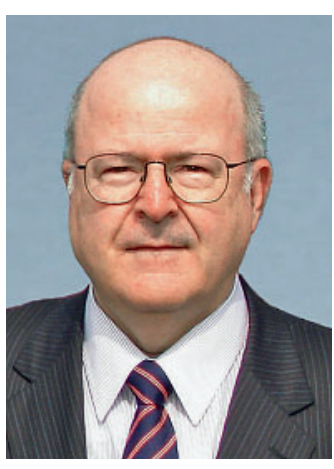

Werner Bauer
La vie de tout individu et de tout groupe - comme le corps médical - est rythmée par des phases de progression, de transformation mais aussi par des phases, plus difficiles, de remise en question.

Quiconque prend le temps de discuter avec des médecins, de lire des lettres de lecteurs ou des comptes rendus de congrès se rend vite compte que le progrès ne fait de loin pas l'unanimité au sein du corps médical. Il est vrai que, dans de nombreux domaines diagnostiques et thérapeutiques, la médecine va de succès en succès; mais la profession dans son ensemble ne parvient pas à s'en réjouir. Enracinés dans la société actuelle, les médecins sont également confrontés aux problèmes de cette dernière: partage des ressources, croissance démographique, pluralité des idéologies. Qui dit nouveaux traitements et nouveaux médicaments dit nouvelles questions sur les coûts, sur l'utilité marginale et sur la pose de l'indication. Les considérations économiques, politiques et éthiques sont autant de freins à l'esprit pionnier et à la volonté de progresser des médecins.

Nul ne peut échapper aux bouleversements que traversent actuellement notre système de santé et notre profession. Pourtant, bien peu de médecins considèrent ces transformations rapides des conditions et des structures de travail comme un défi et une chance - ils ne sont d'ailleurs pas une exception au sein de la population.

La différence est considérable entre un groupe qui a l'impression de participer activement aux changements et un groupe qui au contraire a l'impression d'être à la merci des évolutions et des nouveautés. Bien souvent, le deuxième groupe réagira en se raccrochant au passé, en combattant les nouveautés ou du moins en essayant de ralentir encore un peu l'arrivée «du pire». Difficile de mettre un nom sur le moteur de ces changements: est-ce «l'Etat», «les politiciens», «les caisses-maladie», «les économistes», «l'air du temps», «l'Europe»? Peu importe - combien de fois nous sommes-nous entendus dire, à juste titre, que nous aurions dû réagir plus tôt, et qu'il ne nous reste maintenant pas d'autre solution que le retrait ou la dérobade?

Les médecins ont peur de remettre en question des mesures qui ont fait leurs preuves. Pour eux, le processus de transformation dont ils sont témoins mène inexorablement à la déprofessionnalisation de leur profession. Une profession «déprofessionnalisée» est une profession dont le destin, de la formation aux structures de travail en passant par la définition des exigences en matière de qualité et par la rémunération n'est en grande partie plus entre les mains des personnes qui l'exercent.

Et c'est précisément ce sentiment que partagent de nombreux médecins. Ce sentiment de ne plus être maîtres de leur destin, et cette peur de voir des valeurs médicales fondamentales se perdre et la relation privilégiée qu'ils partagent avec les patients se dégrader. Coresponsabilité économique, réduction des indications, rationalisation cachée, "patient transparent», nouveaux modèles de financement et forfaits par cas autant de changements qui suscitent la méfiance des médecins. Les médecins de famille ne sont pas épargnés, pas plus que les médecins hospitaliers, qui voient l'introduction du système DRG s'approcher avec appréhension.

Et maintenant? Que cherchons-nous? De la compassion?

Bien sûr que non. D'abord, nous n'en voulons pas; ensuite, cela ne nous serait d'aucune utilité. Même si nous nourrissons l'espoir secret que nos efforts trouvent de la sympathie auprès de la population, des politiciens et des journalistes et que ceux-ci nous soutiennent, le corps médical ne doit se faire aucune illusion. Il est vrai que les médecins obtiennent toujours d'excellents résultats dans les sondages de confiance et de popularité des professions, mais c'est tout. Si les médecins ne prennent pas rapidement leur destin en main, en faisant preuve de créativité, d'initiative et de détermination, en étant prêt à accepter les changements, et en usant habilement de leur influence - influence qu'ils n'ont pas encore perdue dans les processus décisionnels, aucune compassion ne peut les aider. Pour sauver notre profession, nous devons agir maintenant, et ne pas attendre qu'il soit trop tard!

Je ne peux terminer ce «call for action» sans cette citation, qui m'avait alors inspiré un précédent «Et encore» et qui est aujourd'hui encore plus d'actualité: «The future is not a place like the Isle of Wight awaiting our arrival. It is more like the great Western Railway, something that we have to imagine, design and build. If we do not build it other people will!» [1].

Werner Bauer*

1 Royal College of Physicians of London: Future Physicians - changing doctors in changing times; 2010. 